\title{
Corrigendum
}

\section{Corrigendum to "Performance Investigation of Tunnel Lining with Cavities around Surrounding Rocks"}

\author{
Jiajia Li, ${ }^{1,2,3}$ Yong Fang $\left(\mathbb{D},{ }^{2}\right.$ Cheng Liu, ${ }^{3}$ Yongxing Zhang $\mathbb{D}^{1,2,3}$ and Weihua Lu $\mathbb{D}^{3}$ \\ ${ }^{1}$ Hunan Provincial Key Laboratory of Hydropower Development Key Technology, Changsha 410014, China \\ ${ }^{2}$ Key Laboratory of Transportation Tunnel Engineering (Southwest Jiaotong University), Ministry of Education, \\ Chengdu 610031, China \\ ${ }^{3}$ Nanjing Forestry University, Nanjing 210037, China
}

Correspondence should be addressed to Yongxing Zhang; zhanguongxing81@aliyun.com

Received 5 August 2021; Accepted 5 August 2021; Published 24 August 2021

Copyright ( 2021 Jiajia Li et al. This is an open access article distributed under the Creative Commons Attribution License, which permits unrestricted use, distribution, and reproduction in any medium, provided the original work is properly cited.

In the article titled "Performance Investigation of Tunnel Lining with Cavities around Surrounding Rocks" [1], authors Cheng Liu and Weihua Lu were affiliated to "Nanjing Forestry University, Hunan Provincial Key Laboratory of Hydropower Development Key Technology, Nanjing, China," which is incorrect. The correct affiliations for this author are as follows: Nanjing Forestry University, Nanjing 210037, China.

The corrected list of affiliations is shown in the author information above.

\section{References}

[1] J. Li, Y. Fang, C. Liu, Y. Zhang, and W. Lu, "Performance Investigation of Tunnel Lining with Cavities around Surrounding Rocks," Advances in Civil Engineering, vol. 2020, Article ID 1364984, 5 pages, 2020. 